\title{
Comentario a: "Linfadenectomía retroperitoneal laparoscópica primaria para el tumor testicular de células germinales no seminomatoso en estadio clínico l"
}

\author{
A. Alcaraz \\ Departamento de Urología, Hospital Clínic, Barcelona, España
}

Accesible en línea el 5 de enero de 2011

En el presente número de Actas Urológicas Españolas, encontramos un importante trabajo realizado por Castillo et al en el cual nos muestran la posibilidad de realizar la linfadenectomía retroperitoneal de forma laparoscópica como tratamiento primario del cáncer de testículo en estadio ${ }^{1}{ }^{1}$. La importancia de este estudio radica en ser la mayor serie publicada y en que los pacientes han sido operados por un cirujano de amplia experiencia oncológica y laparoscópica. Dos son los aspectos que creemos merecen debate en el presente comentario editorial: en primer lugar, la indicación de la linfadenectomía como tratamiento primario y, en segundo lugar, el papel que desempeña actualmente la cirugía laparoscópica en su intento de suplantar a la cirugía abierta en las linfadenectomías retroperitoneales, no tan sólo en el estadio I, sino en el rescate de las masas posquimioterapia ${ }^{2}$.

Existen dos tendencias en el manejo del estadio I. La linfadenectomía retroperitoneal, ampliamente extendida en EE.UU. y Latinoamérica, exige una gran cirugía (especialmente agresiva si se realiza en abordaje abierto). Tiene como objetivo diagnosticar el $20 \%$ de pacientes con tumor viable en el retroperitoneo, y de esta forma seleccionar los pacientes que recibirán quimioterapia adyuvante, así como evitar futuros crecimientos de restos de teratoma. Entre sus indicaciones claras estaría la imposibilidad o dificultad para el seguimiento con imagen y marcadores de estos pacientes. La segunda alternativa, ampliamente extendida en Europa, es el tratamiento expectante en

Véase contenido relacionado en

DOI:10.1016/j.acuro.2010.08.004.

Correo electrónico: aalcaraz@clinic.ub.es pacientes de bajo riesgo y el tratamiento con dos ciclos de quimioterapia (BEP) en aquellos de alto riesgo de metástasis retroperitoneales, que son los pacientes con carcinoma embrionario o invasión linfovascular en el tumor primario. Con este abordaje, es conocido que se está sobretratando al menos a la mitad de los pacientes, pero evitamos un número muy importante de linfadenectomías retroperitoneales sin que exista repercusión en la mortalidad.

En cuanto a si la laparoscopia puede sustituir a la cirugía abierta, existen evidencias de que el número de recurrencia es similar en ambas cirugías cuando son realizadas por cirujanos con experiencia ${ }^{3}$, permitiendo reducir de forma sustancial la morbilidad de esta cirugía, manteniendo las cifras de supervivencia. Ha ayudado a reducir esta morbilidad, en especial la eyaculación retrógrada, el empleo de las plantillas (templates) retroperitoneales unilaterales, que sin duda deben ser empleadas en el estadio I; incluso existen evidencias de su seguridad para emplearse en el rescate de masas residuales ${ }^{4}$.

En conclusión, siguen existiendo las dos tendencias de tratamiento de estadio I, la linfadenectomía tendrá su lugar en especial en pacientes en los que no podemos asegurar su seguimiento, y en caso de tener que realizarse, la laparoscopia es el procedimiento menos mórbido. Pero sin duda, estos pacientes deberán ser operados en centros de amplia experiencia.

\section{Bibliografía}

1. Castillo OA, Sánchez-Salas R, Secin FP, Campero JM, Foneron A, Vidal-Mora I. Linfadenectomía retroperitoneal laparoscópica 
primaria para tumor germinal no seminomatoso en estadio clínico I. Actas Urol Esp. 2011;35:22-8.

2. Katz MH, Eggener SE. The evolution, controversias and potencial pitfalls of modified retroperitoneal lymph node dissection templates. World J Urol. 2009;27:477-83.

3. Rassweiler JJ, Scheitlin W, Heidenreich A, Laguna MP, Janetschek G. Laparoscopic retroperitoneal lymph node dissection: does it still have a role in the management of clinical stage I nonseminomatous testis cancer? A European perspective. Eur Urol. 2008;54:1004-15.

4. Heidenreich A, Pfister D, Witthuhn R, Thüer D, Albert P. Postchemotherapy retroperitoneal lymph node dissection in advanced testicular cancer: radical or modified template resection. Eur Urol. 2009;55:217-24.

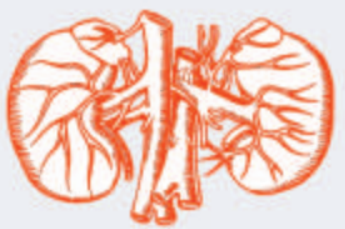

\section{PROGRAMA FORMATIVO ACTAS UROLÓGICAS ESPAÑOLAS 2011 EXAMEN DE ENERO 2011 / VOLUMEN 35 NÚMERO 1}

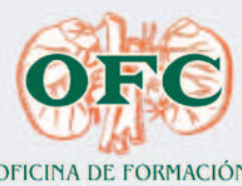
CONTINUADA

Los artículos seleccionados como lecturas recomendadas en este número de Actas Urológicas Españolas son:

Linfadenectomía retroperitoneal laparoscópica primaria para el tumor testicular de células germinales no seminomatoso en estadio clínico I. Castillo OA, Sánchez-Salas R, Secin FP, Campero JM, Foneron A, Vidal-Mora I.

Comentario a "Linfadenectomía retroperitoneal laparoscópica como tratamiento primario del tumor de testículo de células germinales no seminomatoso en estadio I". Alcaraz A.

Retransplante renal: factores de riesgo y resultados. Arce J, Rosales A, Caffaratti J, Montlleó M, Guirado L, Díaz JM, Villavicencio H.

Experiencia con sunitinib en cáncer de próstata metastásico hormonorresistente sin respuesta a docetaxel. Gasent JM, Grande E, Casinello J, Provencia M, Laforga JB, Alberola V.

El cuestionario se completa y envía on-line a través de la WEB del programa http://www.aeu.es/pfaue2011/

Con el $100 \%$ de respuestas acertadas se consiguen 0.4 créditos por número, que equivalen a 2 horas lectivas.

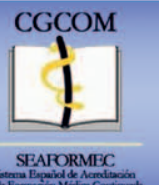

Actividad acreditada en base a la encomienda de gestión concedida por los Ministerios de Sanidad y Política Social y Ministerio de Educación al Consejo General de Colegios Oficiales de Médicos (Resolución de 22 de marzo de 2002, BOE Num. 81 de 4 de abril de 2002) con 0,4 CRÉDITOS, equivalentes a $\mathbf{2}$ horas lectivas

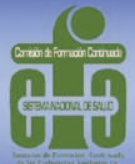

